\title{
BIDSonym - a BIDSapp for the pseudo-anonymization of neuroimaging datasets
}

\author{
Peer Herholz ${ }^{1, *}$, Rita Marie Ludwig ${ }^{2}$, Jean-Baptiste Poline ${ }^{1}$ \\ ${ }^{1}$ NeuroDataScience-Origami lab, McConnell Brain Imaging Centre, \\ The Neuro (Montreal Neurological Institute-Hospital), \\ Faculty of Medicine, McGill University, Montreal, Quebec, Canada \\ ${ }^{2}$ University of Oregon, Eugene, United States \\ *Correspondence to: herholz.peer@gmail.com
}

\begin{abstract}
The amount of neuroimaging data being shared increased exponentially in recent years. While this development introduces prominent advantages concerning open, reproducible and sustainable neuroimaging, the process of data sharing must ensure the privacy of participant data. A requirement from both, Ethics Review Boards and data sharing resources, datasets need to be (pseudo-) anonymized prior to sharing in order to limit participant re-identification. Depending on the dataset at hand, this process can however become cumbersome and prone to errors. Here we introduce BIDSonym, a tool for automated pseudo-anonymization of neuroimaging datasets. BIDSonym supports multiple de-identification procedures and operates on neuroimaging, as well as metadata files. In addition, all metadata information present in the respective files is gathered and evaluated. Its outputs furthermore allow users to conduct a more in-depth assessment of potentially sensitive information present in a given dataset. Through its workflow and utilization of the Brain Imaging Data Structure (BIDS), BIDSonym's application is reproducible, requires no manual intervention and is agnostic to idiosyncrasies of small and large scale datasets.

Keywords: data pseudo-anonymization | neuroimaging datasets | de-identification | data sharing
\end{abstract}

\section{INTRODUCTION}

Due to the evolution of research incentives, technical advancements and the development of new standards (Eickhoff et al. 2016, Gorgolewski et al. 2016, Nichols et al. 2017, Poldrack et al. 2013, Poldrack and Gorgolewski 2014, 2017), increasingly greater amounts of neuroimaging data are being shared either publicly or made available through data user agreements. These datasets originate from small samples of participants collected by individual research groups, as well as from "Big Data" samples including thousands of participants collected by large research consortia (UK Biobank (Sudlow et al. 2015), HCP (Van Essen et al. 2013), ABIDE (Di Martino et al. 2014), ADNI (Mueller et al. 2005), etc.). While data sharing is important and beneficial (Eickhoff et al. 2016, Nichols et al. 2017, Poldrack and Gorgolewski 2014, Poline et al. 2012), privacy of participant data must be protected (Bannier et al. 2020, Brakewood and Poldrack 2013). To that end, Ethic Review Boards and data sharing platforms typically require that uploaded datasets are provided in anonymized or pseudo-anonymized form, limiting participant reidentification. However, the (pseudo-) anonymization process is deceptively complex; attempts at ensuring data privacy must take into consideration all dataset components, including imaging modalities, as well as national legal and ethical frameworks. Several algorithms have been developed to (pseudo-) anonymise imaging datasets but they offer limited solutions. Some are attached to specific software, some are limited to specific computing environments; most miss an in-depth assessment and treatment of the metadata attached to the dataset, or lack the capacity to automatize anonymization across large datasets. BIDSonym was created to ad- dress these points in one simple, flexible, and general tool that offers users an array of automated (pseudo-) anonymization options to augment participant privacy in neuroimaging datasets.

There are two components of neuroimaging datasets that arguably pose the largest risk to maintaining participant privacy: the structural images and accompanying metadata (e.g. metadata text files or information embedded in image file headers). Structural images contain visible identifiable participant information via facial features like the eyes, nose, and mouth, and privacy is usually addressed through a process called "defacing" within which all or a subset of these features are removed from the final structural data files. The metadata text files may additionally contain identifiable participant data through the recording of acquisition time and location, and personal details such as date of birth, height, and weight. Here, privacy is maintained by removing or blurring this information from the final dataset. BIDSonym addresses both vulnerabilities in neuroimaging datasets, obviating the need for multiple steps within a data sharing pipeline to ensure participant privacy.

\section{BIDSonym: ARCHITECTURE \& FUNCTIONALITY}

In concordance with the BIDS-App template (Gorgolewski et al. 2017), BIDSonym operates as a command line tool written in Python (Rossum 1995) and is intended to run in its containerized version (either using Docker (https://www.docker.com) or Singularity (https://sylabs.io), providing all necessary software dependencies. However, it is also available as a Python package via pypi (https://pypi.org) to facilitate reuse in a development environment. BIDSonym expects BIDS datasets (Gorgolewski et al. 2016) and provides three 


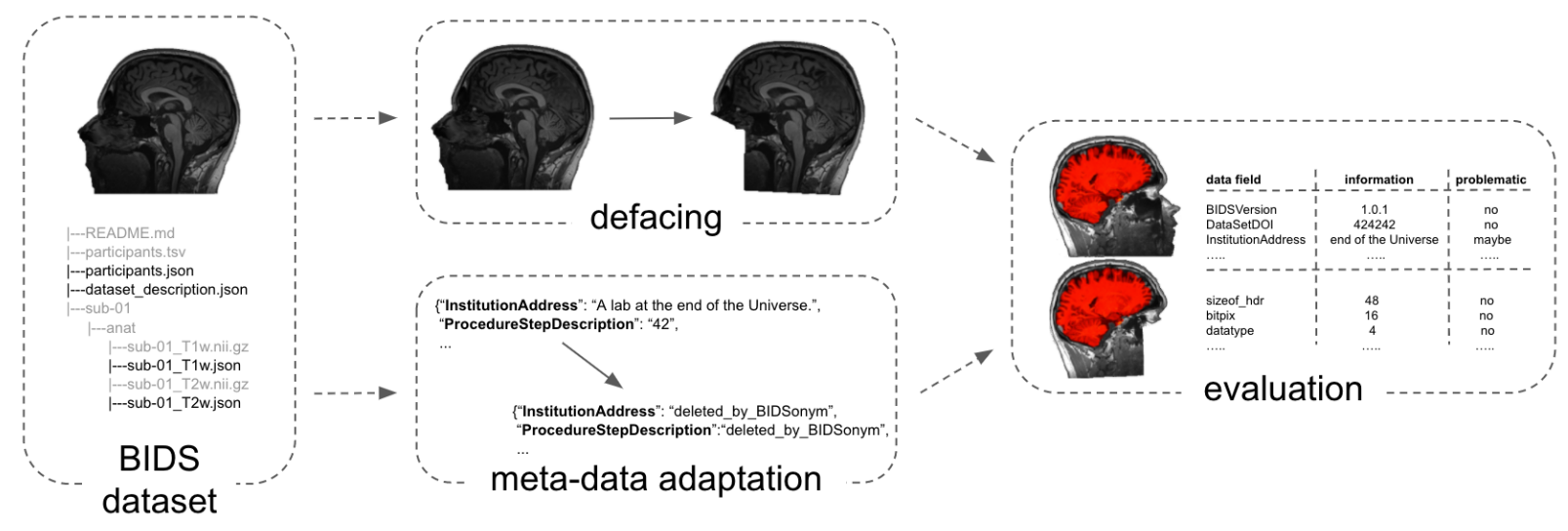

Figure 1. Overview of BIDSonym's functionality | Providing a dataset in BIDS as input, structural images are defaced, meta-data fields adapted as requested and the performance of the defacing, as well as all meta-data fields (in both the json sidecar files and image headers) evaluated.

core functionalities as depicted in Figure 1: defacing of structural (i.e. T1 and T2 weighted images, adaptation of potentially sensitive metadata information, and evaluation of (pseudo-) anonymization results.

Focusing on the first aspect, BIDSonym supports a multitude of commonly used defacing algorithms and tools, including pydeface (Gulban et al. 2019), mri deface (Bischoff-Grethe et al. 2007), quickshear (Schimke and Hale 2011), and mridefacer (Hanke and Halchenko 2018). Based on the chosen tool, facial features of the structural images of either specified participants or the whole data set are then removed. Figure 2 provides an example for the different algorithms and tools available for defacing a structural T1 weighted image. Furthermore, structural images from other modalities (e.g. T2 weighted) can also be defaced in which case the defaced T1 weighted structural image will be utilized as a deface mask. In order to account for possible errors during the defacing process, BIDSonym moves the non-defaced original structural images into a distinct directory before defacing, allowing users to test multiple defacing settings and/or options. Following BIDS (Gorgolewski et al. 2016), those files are moved to a different directory ('/sourcedata') and a description identifier ('*_descnondeid $\left.{ }^{*}\right)$ is added to the filenames.

A comparable behavior is implemented with regard to metadata information in BIDSonym's second core functionality. The information in the metadata files that accompany neuroimaging data and in the headers of neuroimaging data files will be gathered and listed within a tabular file ('*.tsv'). If specified, the extracted information will then be queried for potentially sensitive information (e.g. name, date or place of birth, etc.) and marked accordingly. Additionally, users can specify that certain information should be deleted from metadata files, in which case they will be moved and renamed as described for the neuroimaging data.

BIDSonym's third core functionality implements quality control assessments of the defacing results and of the information present in the data. Concerning the first, this includes (interactive) plots that allow to evaluate if the applied algorithm and settings were too stringent (e.g. removing voxels belonging to the brain). Regarding the second, information present in the meta-data files and image headers are gathered in tabular format within respective files ('*.tsv'). Each table contains all key-value pairs present in a given file and aims to provide an assessment of potentially sensitive information.

\section{SUMMARY}

More information on BIDSonym's workflow, the corresponding processing steps and outcomes, as well as installation instructions can be found in the respective documentation (https: //peerherholz.github.io/BIDSonym) and GitHub repository (https://github.com/PeerHerholz/BIDSonym).

BIDSonym provides a straightforward and flexible way to pseudo-anonymize neuroimaging datasets by a variety of means, operating on both small and large datasets through its implementation following the BIDS-App template (Gorgolewski et al. 2017). BIDSonym depends on the nibabel (Brett et al. 2020), nipype (Gorgolewski et al. 2011), nilearn (Abraham et al. 2014), pybids (Yarkoni et al. 2019) and pandas (McKinney 2010) python packages (all are well maintained and tested) and is licensed under the BSD-3 license (https://opensource. org/licenses/BSD-3-Clause). As data sharing becomes more widely adopted, BIDSonym fills an important gap for the neuroimaging community. 


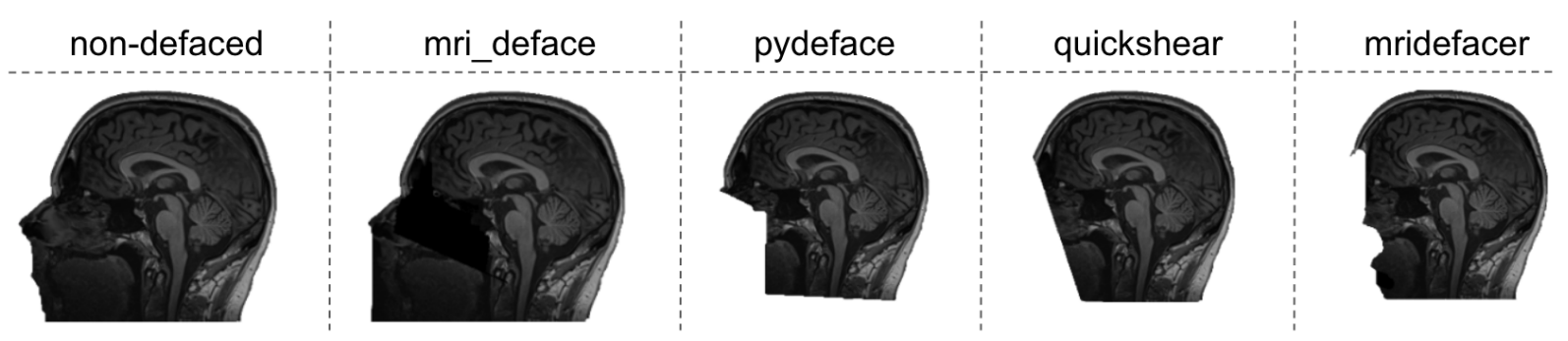

Figure 2. Defacing examples | Results of the different algorithms and tools (columns) included in BIDSonym, displayed in comparison to the corresponding original structural image (most left).

\section{ACKNOWLEDGEMENTS}

P.H. and J.B.P. were supported in parts by funding from the Canada First Research Excellence Fund, awarded to McGill University for the Healthy Brains for Healthy Lives initiative, the National Institutes of Health (NIH) NIH-NIBIB P41 EB019936 (ReproNim), as well as the National Institute Of Mental Health of the NIH under Award Number R01MH096906. P.H. was additionally supported by research scholar award from Brain Canada, in partnership with Health Canada, for the Canadian Open Neuroscience Platform initiative. This project originated as part of Neurohackademy which is funded by the National Institute of Mental Health through a grant to Ariel Rokem and Tal Yarkoni (R25MH112480). Finally, all the contributors listed in the project's Zenodo and GitHub repository have contributed code and intellectual labor to further improve BIDSonym. The same holds true for users that reported issues and continue to do so.
Abraham, A., Pedregosa, F., Eickenberg, M., Gervais, P., Mueller, A., Kossaifi, J., Gramfort, A., Thirion, B., and Varoquaux, G. (2014). Machine learning for neuroimaging with scikit-learn. Frontiers in Neuroinformatics, 8. Publisher: Frontiers.

Bannier, E., Barker, G., Borghesani, V., Broeckx, N., Clement, P., Vaya, M. d. 1. I., Emblem, K. E., Ghosh, S., Glerean, E., Gorgolewski, K. J., Havu, M., Halchenko, Y. O., Herholz, P., Hespel, A., Heunis, S., Hu, Y., Chuan-Peng, H., Huijser, D., Jancalek, R., Dr. Vasileios K. Katsaros, M. D., Kieseler, M.-L., Maumet, C., Moreau, C., Mutsaerts, H. J., Oostenveld, R., Isik, E. O., Espinosa, N. P. L., Pellman, J., Pernet, D. C., Pizzini, F. B., Trbalić, A. , Toussaint, P.-J., Castello, M. V. d. O., Wang, F., Wang, C., and Zhu, H. (2020). The Open Brain Consent: Informing research participants and obtaining consent to share brain imaging data. Technical report, PsyArXiv.

Bischoff-Grethe, A., Ozyurt, I. B., Busa, E., Quinn, B. T., Fennema-Notestine, C., Clark, C. P., Morris, S., Bondi, M. W., Jernigan, T. L., Dale, A. M., Brown, G. G., and Fischl, B. (2007). A Technique for the Deidentification of Structural Brain MR Images. Human brain mapping, 28(9):892-903.

Brakewood, B. and Poldrack, R. A. (2013). The ethics of secondary data analysis: Considering the application of Belmont principles to the sharing of neuroimaging data. NeuroImage, 82:671-676.

Brett, M., Markiewicz, C. J., Hanke, M., Côté, M.-A., Cipollini, B., McCarthy, P., Jarecka, D., Cheng, C. P., Halchenko, Y. O., Cottaar, M., Ghosh, S., Larson, E., Wassermann, D., Gerhard, S., Lee, G. R., Wang, H.-T., Kastman, E., Kaczmarzyk, J., Guidotti, R., Duek, O., Rokem, A., Madison, C., Morency, F. C., Moloney,
B., Goncalves, M., Markello, R., Riddell, C., Burns, C., Millman, J., Gramfort, A., Leppäkangas, J., Sólon, A., van den Bosch, J. J., Vincent, R. D., Braun, H., Subramaniam, K., Gorgolewski, K. J., Raamana, P. R., Nichols, B. N., Baker, E. M., Hayashi, S., Pinsard, B., Haselgrove, C., Hymers, M., Esteban, O., Koudoro, S., Oosterhof, N. N., Amirbekian, B., Nimmo-Smith, I., Nguyen, L., Reddigari, S., St-Jean, S., Panfilov, E., Garyfallidis, E., Varoquaux, G., Legarreta, J. H., Hahn, K. S., Hinds, O. P., Fauber, B., Poline, J.-B., Stutters, J., Jordan, K., Cieslak, M., Moreno, M. E., Haenel, V., Schwartz, Y., Baratz, Z., Darwin, B. C., Thirion, B., Papadopoulos Orfanos, D., Pérez-García, F., Solovey, I., Gonzalez, I., Palasubramaniam, J., Lecher, J., Leinweber, K., Raktivan, K., Fischer, P., Gervais, P., Gadde, S., Ballinger, T., Roos, T., Reddam, V. R., and freec84 (2020). nibabel.

Di Martino, A., Yan, C.-G., Li, Q., Denio, E., Castellanos, F. X., Alaerts, K., Anderson, J. S., Assaf, M., Bookheimer, S. Y., Dapretto, M., Deen, B., Delmonte, S., Dinstein, I., Ertl-Wagner, B., Fair, D. A., Gallagher, L., Kennedy, D. P., Keown, C. L., Keysers, C., Lainhart, J. E., Lord, C., Luna, B., Menon, V., Minshew, N. J., Monk, C. S., Mueller, S., Müller, R.-A., Nebel, M. B., Nigg, J. T., O'Hearn, K., Pelphrey, K. A., Peltier, S. J., Rudie, J. D., Sunaert, S., Thioux, M., Tyszka, J. M., Uddin, L. Q., Verhoeven, J. S., Wenderoth, N., Wiggins, J. L., Mostofsky, S. H., and Milham, M. P. (2014). The autism brain imaging data exchange: towards a large-scale evaluation of the intrinsic brain architecture in autism. Molecular Psychiatry, 19(6):659-667.

Eickhoff, S., Nichols, T. E., Van Horn, J. D., and Turner, J. A. (2016). Sharing the wealth: Neuroimaging data repositories. NeuroImage, 124(Pt B):1065-1068.

Gorgolewski, K. J., Alfaro-Almagro, F., Auer, T., Bellec, P., Capotă, M., Chakravarty, M. M., Churchill, N. W., Cohen, 
A. L., Craddock, R. C., Devenyi, G. A., Eklund, A., Esteban, O., Flandin, G., Ghosh, S. S., Guntupalli, J. S., Jenkinson, M., Keshavan, A., Kiar, G., Liem, F., Raamana, P. R., Raffelt, D., Steele, C. J., Quirion, P.-O., Smith, R. E., Strother, S. C., Varoquaux, G., Wang, Y., Yarkoni, T., and Poldrack, R. A. (2017). BIDS apps: Improving ease of use, accessibility, and reproducibility of neuroimaging data analysis methods. PLOS Computational Biology, 13(3):e1005209.

Gorgolewski, K. J., Auer, T., Calhoun, V. D., Craddock, R. C., Das, S., Duff, E. P., Flandin, G., Ghosh, S. S., Glatard, T., Halchenko, Y. O., Handwerker, D. A., Hanke, M., Keator, D., Li, X., Michael, Z., Maumet, C., Nichols, B. N., Nichols, T. E., Pellman, J., Poline, J.-B., Rokem, A., Schaefer, G., Sochat, V., Triplett, W., Turner, J. A., Varoquaux, G., and Poldrack, R. A. (2016). The brain imaging data structure, a format for organizing and describing outputs of neuroimaging experiments. Scientific Data, 3(1):1-9.

Gorgolewski, K. J., Burns, C. D., Madison, C., Clark, D., Halchenko, Y. O., Waskom, M. L., and Ghosh, S. S. (2011). Nipype: A Flexible, Lightweight and Extensible Neuroimaging Data Processing Framework in Python. Frontiers in Neuroinformatics, 5.

Gulban, O. F., Nielson, D., Poldrack, R., Lee, J., Gorgolewski, K. J., Sochat, V., and Ghosh, S. (2019). pydeface.

Hanke, M. and Halchenko, Y. (2018). mridefacer. original-date: 2015-09-21T15:25:14Z.

McKinney, W. (2010). Data structures for statistical computing in python. In Proceedings of the 9th Python in Science Conference, volume 445, pages 51-56.

Mueller, S. G., Weiner, M. W., Thal, L. J., Petersen, R. C., Jack, C., Jagust, W., Trojanowski, J. Q., Toga, A. W., and Beckett, L. (2005). The Alzheimer's Disease Neuroimaging Initiative. Neuroimaging Clinics of North America, 15(4):869-877.

Nichols, T. E., Das, S., Eickhoff, S. B., Evans, A. C., Glatard, T., Hanke, M., Kriegeskorte, N., Milham, M. P., Poldrack, R. A., Poline, J.-B., Proal, E., Thirion, B., Van Essen, D. C., White, T., and Yeo, B. T. T. (2017). Best practices in data analysis and sharing in neuroimaging using MRI. Nature Neuroscience, 20(3):299-303.

Poldrack, R. A., Barch, D. M., Mitchell, J., Wager, T., Wagner, A. D., Devlin, J. T., Cumba, C., Koyejo, O., and Milham, M. (2013). Toward open sharing of task-based fMRI data: the OpenfMRI project. Frontiers in Neuroinformatics, 7.

Poldrack, R. A. and Gorgolewski, K. J. (2014). Making big data open: data sharing in neuroimaging. Nature Neuroscience, 17(11):1510-1517.

Poldrack, R. A. and Gorgolewski, K. J. (2017). OpenfMRI: Open sharing of task fMRI data. NeuroImage, 144:259-261.

Poline, J.-B., Breeze, J. L., Ghosh, S. S., Gorgolewski, K. J., Halchenko, Y. O., Hanke, M., Helmer, K. G., Marcus, D. S., Poldrack, R. A., Schwartz, Y., Ashburner, J., and Kennedy, D. N. (2012). Data sharing in neuroimaging research. Frontiers in Neuroinformatics, 6.

Rossum, G. (1995). Python reference manual. Technical Report, CWI (Centre for Mathematics and Computer Science), NLD.
Schimke, N. and Hale, J. (2011). Quickshear defacing for neuroimages. In Proceedings of the 2nd USENIX conference on Health security and privacy, HealthSec'11, page 11, USA. USENIX Association.

Sudlow, C., Gallacher, J., Allen, N., Beral, V., Burton, P., Danesh, J., Downey, P., Elliott, P., Green, J., Landray, M., Liu, B., Matthews, P., Ong, G., Pell, J., Silman, A., Young, A., Sprosen, T., Peakman, T., and Collins, R. (2015). UK Biobank: An Open Access Resource for Identifying the Causes of a Wide Range of Complex Diseases of Middle and Old Age. PLoS Medicine, 12(3).

Van Essen, D. C., Smith, S. M., Barch, D. M., Behrens, T. E. J., Yacoub, E., and Ugurbil, K. (2013). The WU-Minn Human Connectome Project: An overview. NeuroImage, 80:62-79.

Yarkoni, T., Markiewicz, C. J., Vega, A. d. 1., Gorgolewski, K. J., Salo, T., Halchenko, Y. O., McNamara, Q., DeStasio, K., Poline, J.-B., Petrov, D., Hayot-Sasson, V., Nielson, D. M., Carlin, J., Kiar, G., Whitaker, K., DuPre, E., Wagner, A., Tirrell, L. S., Jas, M., Hanke, M., Poldrack, R. A., Esteban, O., Appelhoff, S., Holdgraf, C., Staden, I., Thirion, B., Kleinschmidt, D. F., Lee, J. A., Castello, M. V. O. d., Notter, M. P., and Blair, R. (2019). PyBIDS: Python tools for BIDS datasets. Journal of Open Source Software, 4(40):1294. 